\title{
A MULTI-WAVELENGTH STUDY OF X-RAY SELECTED AGN
}

\author{
R. J. V. BRISSENDEN ${ }^{1}$, I. R. TUOHY 1 , G. V. BICKNELL ${ }^{1}$, \\ R. A. REMILLARD ${ }^{2}$ and D. A. SCHWARTZ ${ }^{3}$ \\ ${ }^{1}$ Mt. Stromlo and Siding Spring Observatories, the Australian National \\ University, Private Bag, PO Woden, ACT 2606, Australia \\ ${ }^{2}$ Center for Space Research, MIT, Cambridge, MA 02139 \\ ${ }^{3}$ Harvard-Smithsonian Cf A, 60 Garden Street, Cambridge, MA 02138
}

\section{Observations}

A sample of Active Galactic Nuclei (AGN) have been discovered during a program to identify the optical counterparts of X-ray sources detected by the Modulation Collimator experiment of the High Energy Astronomy Observatory-1 (HEAO-1). UV-excess techniques were used to identify the X-ray sources (Remillard et al. 1986) and the details of the identifications are given elsewhere (Remillard et al. 1988, Brissenden et al. 1988). We report here the preliminary results of a multi-wavelength study of these new AGN.

The majority of the sample, comprised of 17 Seyfert 1 galaxies, 5 BL Lac objects and 4 QSOs were observed with the VLA during 1988 May 4 or 7, at both $20 \mathrm{~cm}$ and $6 \mathrm{~cm}$ with the C array in "snapshot" mode. Two additional QSOs observed and detected by Parkes at $3.6 \mathrm{~cm}$ and the Fleurs Synthesis Telescope at $20 \mathrm{~cm}$ were also included. All but two Seyfert 1 galaxies and two QSOs were detected at $6 \mathrm{~cm}$ with the VLA (all detections being greater than $5 \sigma$ above the background rms noise). None of the objects were resolved with a typical resolution of 4" $\times 7^{\prime \prime}$ at $6 \mathrm{~cm}$. The mean 6-20 cm spectral index $\alpha\left(\mathrm{fv}_{\mathrm{v}} \propto \mathrm{v}^{-\alpha}\right)$ was flatter for the BL Lac objects (0.2) than the Seyfert 1 galaxies and QSOs (0.7).

\section{Broadband Spectral Indices}

We take continuum points in the X-ray, optical and radio spectral regions at $2 \mathrm{keV}, 2500 \AA$ and $6 \mathrm{~cm}$ respectively and form the indices $\alpha_{\text {ro and }} \alpha_{0 x}$ (Tananbaum et al. 1983). The radio flux at $6 \mathrm{~cm}$ is taken directly from the VLA results except for the two southerly QSOs where the value is interplolated from the $20 \mathrm{~cm}$ and $3.6 \mathrm{~cm}$ data. The optical flux at $2500 \AA$ was calculated from the B magnitude using the zero magnitude calibration of Allen (1973), a galactic reddening correction and an assumed power law between $4400 \AA$ and $2500 \AA$ of $\alpha=1.0$ for the Seyfert 1 galaxies and QSOs and $\alpha=2.0$ for BL Lac objects. The correction for galactic reddening was derived from $A_{V}=0.18 * \operatorname{cosec}(b)$ where $b$ is the galactic latitude (Schmidt 1968), a ratio of total to selective absorption of 3.2 and $\mathrm{A}_{\lambda}=4.09 * \mathrm{E}(\mathrm{B}-\mathrm{V})$ for $\lambda=4400 \AA$ (Seaton 1979). The monochromatic $2 \mathrm{keV} \mathrm{X}$-ray flux was calculated from the 2-10 keV LASS count rate (Wood et al. 1984) using a conversion factor of 0.0021 and 0.0027 per $10^{-11} \mathrm{erg} \mathrm{cm}^{-2} \mathrm{~s}^{-1}$ for Seyfert 1 galaxies (and QSOs) and BL Lac objects respectively. The assumed X-ray power law for Seyfert 1 galaxies and QSOs was $\alpha=0.7$ and for BL Lac objects was $\alpha=1.2$. The data are presented in Figure 1. 


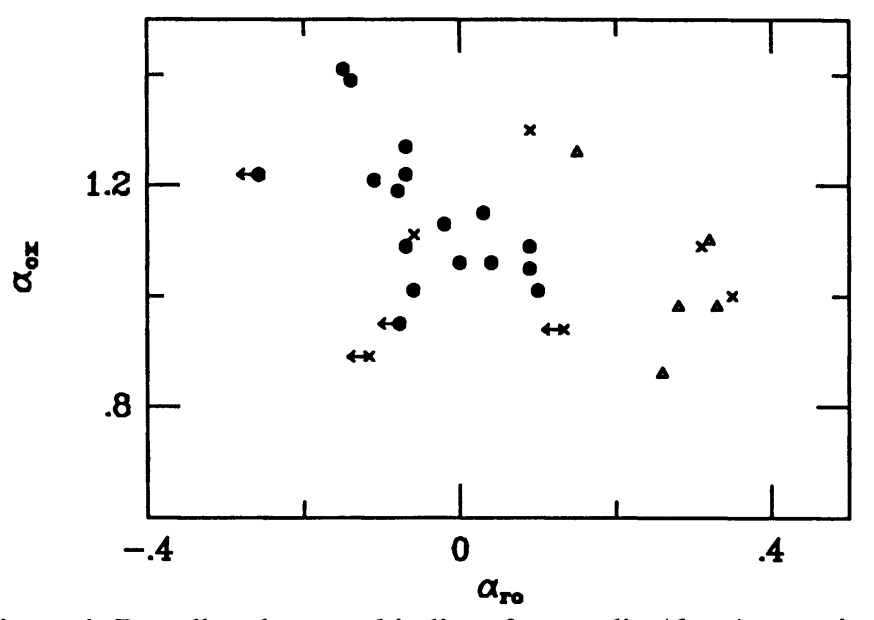

Figure 1. Broadband spectral indices from radio $(6 \mathrm{~cm})$ to optical $(2500 \AA)$ and optical to X-ray $(2 \mathrm{keV})$ continuum points. Seyfert 1 galaxies, QSOs and BL Lac objects are represented by filled circles, crosses and open triangles respectively. $3 \sigma$ upper limits given.

\section{Results and Discussion}

The high detection rate of these X-ray observed AGN with the VLA, allows us to specify their location in the $\alpha_{\text {ro }}-\alpha_{\text {ox }}$ plane. The BL Lac objects lie in a separate, well defined region of Figure $\left.1\left(<\alpha_{\mathrm{ro}}\right\rangle=0.27 \pm 0.07,<\alpha_{\mathrm{ox}}\right\rangle=1.04 \pm 0.15$; the error is the dispersion about the mean) coincident with other X-ray selected BL Lac objects and having flatter overall spectra than radio selected BL Lac objects. The mean redshift of the Seyfert 1 galaxies (QSOs) is $0.05 \pm 0.03(0.19 \pm 0.07)$ with mean $B$ magnitude of $15.67 \pm 0.88$ $(16.41 \pm 0.65)$. They are characterized by very flat optical-radio spectra with $\left\langle\alpha_{\text {ro }}\right\rangle=-0.03 \pm 0.08(0.17 \pm 0.19$; for detections $)$ and steeper optical-X-ray spectral index $\left\langle\alpha_{0 x}\right\rangle=1.15 \pm 0.13(1.06 \pm 0.15)$. The mean values of $\alpha_{\text {ro }}$ and $\alpha_{0 x}$ are very similar to mean values for optically selected Seyfert 1 galaxies (QSOs).We note that the spectral index and luminosity distribution of the present sample coincide with the hard $(2-10 \mathrm{keV})$ selected sample of Piccinotti et al. (1982) but that the mean redshift is higher and mean B magnitude is fainter. Finally, the $2 \mathrm{keV} \mathrm{X}$-ray luminosity and $4.9 \mathrm{GHz}$ radio luminosity are found to correlate for the Seyfert 1 galaxies but no correlation is found between the fluxes. No luminosity correlation is seen for the small number of QSOs and BL Lac objects in the sample.

\section{References}

Allen, C. W. 1973, Astrophysical Quantities, University of London, The Athlone Press. Brissenden, R. J. V., et al. 1988, in preparation.

Piccinotti, G., et al. 1982, Ap. J., 253, 485.

Remillard, R. A., et al. 1986, Ap.J., 301, 742.

Remillard, R. A., et al. 1988, in preparation.

Schmidt, M. 1968, Ap. J., 151, 393.

Seaton, M. J. 1979, M. N. R. A. S., 187, 73P.

Tananbaum, H., Wardle, J. F. C., Zamorani, G., and Anvi, Y. 1983, Ap. J., 268, 60.

Wood, K., et al. 1984, Ap. J. Suppl., 56, 507. 